\title{
Swarm Robotics: The Coordination of Robots via Swarm Intelligence Principles
}

\author{
Marco Dorigo \\ IRIDIA, CoDE, Université Libre de Bruxelles
}

\begin{abstract}
Swarm intelligence is the discipline that deals with natural and artificial systems composed of many individuals that coordinate using decentralized control and self-organization. The discipline focuses on the collective behaviors that result from the local interactions of the individuals with each other and with their environment. Examples of systems studied by swarm intelligence are colonies of ants and termites, schools of fish, and flocks of birds. Some human artifacts also fall into the domain of swarm intelligence. Examples are, among others, some multi-robot systems and certain computer programs tackling optimization and data analysis problems.
\end{abstract}

After a short introduction to swarm intelligence, in my presentation I will focus on recent work in swarm robotics, that is, the application of swarm intelligence principles to the control of swarms of cooperating robots. In particular, I will present results of the swarm-bot experiment. A swarm-bot is an artifact composed of a swarm of assembled s-bots, mobile robots capable of connecting to, and disconnecting from, each other. In the swarm-bot form, the s-bots are attached to each other and, when needed, become a single robotic system that can move and change its shape. S-bots have relatively simple sensors and motors and limited computational capabilities. A swarm-bot can solve problems that cannot be solved by s-bots alone.

In the talk, I will briefly describe the s-bots hardware and the methodology followed to develop algorithms for their control. Then I will focus on the capabilities of the swarm-bot robotic system by showing video recordings of some of the many experiments we performed to study coordinated movement, path formation, self-assembly, collective transport, shape formation, and other collective behaviors. 